\title{
COMBINED INVESTIGATION OF LOW-SCALE FRACTURE IN HYDRATED CEMENT ASSESSED BY NANOINDENTATION AND FIB
}

\author{
J. NĚMEČEK ${ }^{*}$, V. HRBEK ${ }^{\dagger}$, L. POLÍVKA ${ }^{\dagger \dagger}$ AND A. JÄGER ${ }^{\ddagger}$ \\ $" \dagger$ Czech Technical University in Prague \\ Prague, Czech Republic \\ e-mail: jiri.nemecek@fsv.cvut.cz, vladimir.hrbek@fsv.cvut.cz \\ ${ }^{\dagger \dagger}$ Laboratory of Nanostructures and Nanomaterials, Institute of Physics of ASCR \\ Prague, Czech Republic \\ e-mail:polivkal@fzu.cz,jager@fzu.cz
}

Key words: Fracture energy, Fracture toughness, Cement, Nanoindentation, Focused ion beam

\begin{abstract}
This paper deals with an assessment of fracture properties of micro-level components of hydrated cement paste. Fracture energy and fracture toughness are studied by means of several experimental techniques at the micrometer scale. Nanoindentation is used as a primary tool for the material characterization. The work includes analysis of fracture mechanisms under the sharp indenter using focused ion beam (FIB) technology combined with scanning electron microscopy (SEM). Usual microscale approaches used in brittle solids are based on the observation of cracks from surface view after indentation. In this work, an energetic approach and decomposition of work of indentation into plastic and other parts is used instead. Based on cross sectioning of indents via FIB, several assumptions are done on the fracture area under the tip and semi-quantitative estimates of the low-scale fracture energy are performed for the cement paste components. Statistical deconvolution is applied to sort out the individual phase fracture properties.
\end{abstract}

\section{INTRODUCTION}

Fracture mechanisms and fracture energy are of central importance for models that deal with an inelastic phenomena in brittle and quasi-brittle materials including cement and concrete. Fracture properties are traditionally assessed on macro-scale from relatively simple laboratory tests (e.g. tension or three-point bending setup) in which specimens fail by a single localized crack crossing throughout the whole specimen. Therefore, the energy calculation is simplified and the fracture energy can be well estimated. As the scale goes down the situation starts to be more complicated since the fracture paths and extent are no longer straightforward. In recent years, nanoindentation has become a standard technique for assessing elastic and plastic properties of individual material phases at scales below one micrometer for a number of materials including cementitious and timedependent ones [1-3]. However, evaluation of fracture properties requires proper knowledge of the exact failure mechanism, crack patterns and crack dimensions.

In nanoindentation experiment, the fracture toughness or critical strain energy release can be determined for brittle solids in which the crack geometry is well estimated from top of the specimen (surface). Different crack types and shapes were classified e.g. by Cook and Pharr [4]. Most of the cracking pattern is based on radial crack system that starts from indent's corners. For example, Lawn et al. [5] derived the semi-empirical fracture toughness relation 
for half-penny cracks in the form

$$
K_{C}=\alpha\left(\frac{E}{H}\right)^{1 / 2} \frac{P}{c^{3 / 2}}
$$

where $K_{c}$ is the fracture toughness, $E$ is Young's modulus, $H$ is hardness, $P$ is the applied load and $c$ is the crack length that can be observed from the surface top view, $\alpha$ is the empirical coefficient that depends on indenter geometry and crack system.

Eq. (1) is only applicable to bulk brittle materials with the given crack geometry which is not the case of either cementitious materials or some metal alloys where multiple cracks are located at the circumference of the indentation imprint and their exact length and depth cannot be readily observed and measured. To overcome this difficulty, semi-quantitative energetic methods that try to decouple the total indentation work into elastic, plastic and other parts have been developed [6]. The total work of indentation can be decomposed as follows

$$
W_{t o t}=W_{e l}+W_{p l}+U_{f r}+U_{c r}+U_{o t h e r}
$$

where $W_{e l}$ is the elastic deformation part, $W_{p l}$ is the work of pure plastic deformation, $U_{f r}$ is the dissipated energy from cracking surfaces, $U_{c r}$ is the creep associated part of the energy and $U_{\text {other }}$ is a work associated with other processes, e.g. heat release (which is neglected further on, i.e. $\left.U_{\text {other }} \rightarrow 0\right)$. $W_{t o t}, U_{c r}$ and $W_{e l}$ terms in Eq. (2) can be deduced from the experimental load-displacement curve in nanoindentation experiment. The calculation of the energies is illustrated in Fig. 1 where a typical loading-holding-unloading nanoindentation curve is displayed (the dashed line). The total energy $W_{t o t}$ can be calculated from Fig.1 as the hatched area under the loading and holding parts $\left(\mathrm{A}_{1}+\mathrm{A}_{2}+\mathrm{A}_{3}\right) ; \mathrm{A}_{3}$ represents the elastic energy $\left(W_{e l}\right)$ part under unloading curve; $\mathrm{A}_{2}$ stands for creep dissipation during holding $\left(U_{c r}\right)$ and $\mathrm{A}_{1}$ shows plastic and fracture portion $W_{p l}+U_{f r}$.

The plastic work needs to be assessed separately. Based on the dimensional analysis Cheng et al. [7] derived the plastic work from the ratio $k=h_{f} / h_{m}$ between the final $\left(h_{f}\right)$, and the maximum indentation depth $\left(h_{m}\right)$, as

$$
\frac{W_{p l}}{W_{t o t}}=1-\frac{1-3 k^{2}+2 k^{3}}{1-k^{2}}
$$

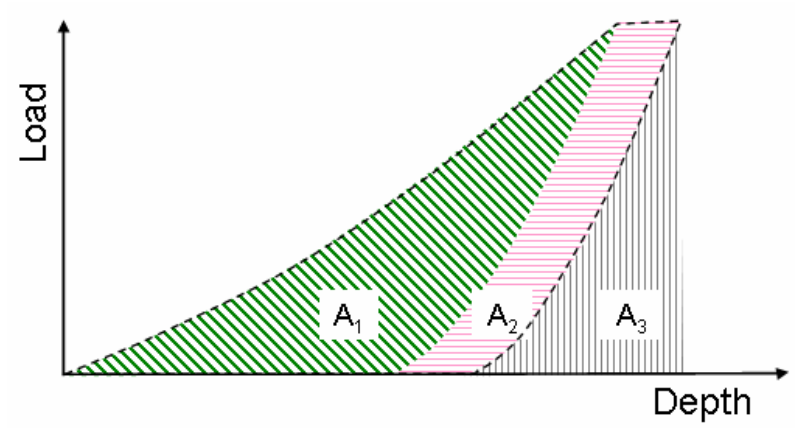

Figure 1: Nanoindentation load-depth diagram (dashed line) and decomposition of energies (hatched areas) after unloading.

Than, the $U_{f r}$ can be assessed and the critical strain energy release rate (fracture energy) computed from

$$
G_{c}=\frac{\partial U_{f r}}{\partial A}=\frac{U_{f r}}{A_{f r}}
$$

in which $A_{f r}$ is the total cracking area.

Since the fracture area is a critical parameter, proper assumptions based on the cracking system must be done. In this work, direct visualization and of the crack system under the indent is provided by focused ion beam milling and scanning electron microscope imaging. The following methodology is applied to assess low-scale parameters of individual hydrated phases in cement paste.

\section{METHODOLOGY}

\subsection{Nanoindentation}

At first, nanoindentation with Berkovich tip was performed on polished samples of well hydrated (1 year) cement paste prepared from cement CEM I-42.5R with water/cement ratio 0.4 . A large number of indents $(\sim 700)$ with an average penetration depth $\sim 200 \mathrm{~nm}$ were performed in several rectangular grids (with 20 micrometer spacing between individual indents) covering statistically representative 
area of the cement microstructure. The loading diagram (Fig. 1) was composed of relatively fast loading (5 s), long holding time (20 s) where most of the creep takes place and unloading (5 s) which is supposed to be elastic.

Then, several representative indents were sectioned by FIB and cracks observed on respective SEM images. Due to very large set of indents it was not possible to inspect every indent individually. However, based on these observations general assumptions on the position, shape and extent of the fracture area was done. The assumptions were used for $G_{c}$ calculation for all of the indents in the grid which led to the statistics of $G_{c}$ appearance. Thus the results can be considered as semiquantitative in this sense but they show well the distribution of the fracture energy between the phases.

Elastic properties were evaluated by standard Oliver and Pharr technique [8] for every indent in a grid. For a conical indentation the reduced modulus is given by

$$
E_{r}=\left.\frac{1}{2 \beta} \frac{\sqrt{\pi}}{\sqrt{A_{c}}} \frac{d P}{d h}\right|_{h=h_{\max }}
$$

where $d P / d h$ is the contact stiffness assessed as the slope of the indentation unloading branch at the maximum depth $h_{\max }, A_{c}$ is the projected contact area of the tip at peak load, and $\beta$ is the geometrical correction factor introduced to correct the non-symmetrical indenter shape $(\beta=1.034$ for the Berkovich tip).

Large number of indents allowed for statistical evaluation and deconvolution of the $E_{r}$ modulus into individual phase distributions (for the deconvolution details see e.g. [1,2]). Due to the deconvolution, indents were separated into groups belonging to distinct material phases, namely the main hydration product (composed mainly of calcium-silicahydrates, C-S-H), calcium hydroxide, nonhydrated clinker minerals and low stiffness (porous) phases. Further, separation of fracture energies was performed into the same groups that were created during deconvolution of $E_{r}$.
In this way, distributions of fracture energies were obtained for distinct material phases.

\subsection{FIB}

Microstructure was analysed with scanning electron microscope combined with focused ion beam FEI Quanta 3D FEG. Cross sections of the indents were revealed by FIB at $30 \mathrm{kV}$ and $100 \mathrm{pA}$. Subsequent SEM observation of the cross sections was carried out at $3 \mathrm{kV}$ and $20 \mathrm{pA}$ as a compromise between the contrast of features and sample charging.

During cross sectioning of the indents part of the sputtered material is redeposited back on a sample surface [9] which can hinder SEM analysis due to pure contrast of details. To prevent this, amorphous $\mathrm{Pt}$ layer with thickness of about $50 \mathrm{~nm}$ was deposited by gas injection system on a surface in the SEM-FIB. This protective layer is visible in Fig. 4. Serial sectioning of the indents was carried out via automated routine of FEI Slice\&View. 50-80 slices with a step of $100 \mathrm{~nm}$ were gained from each indent.

\section{RESULTS AND DISCUSSION}

\subsection{Elastic properties and phase separation}

The statistical deconvolution method gives access to mechanical properties (reduced moduli) and volume fractions for a fixed number of given (normal) distributions. The overall $E_{r}$ distribution and individual phase distributions received after the deconvolution procedure are depicted in Fig. 2 and summarized in Tab. 1.

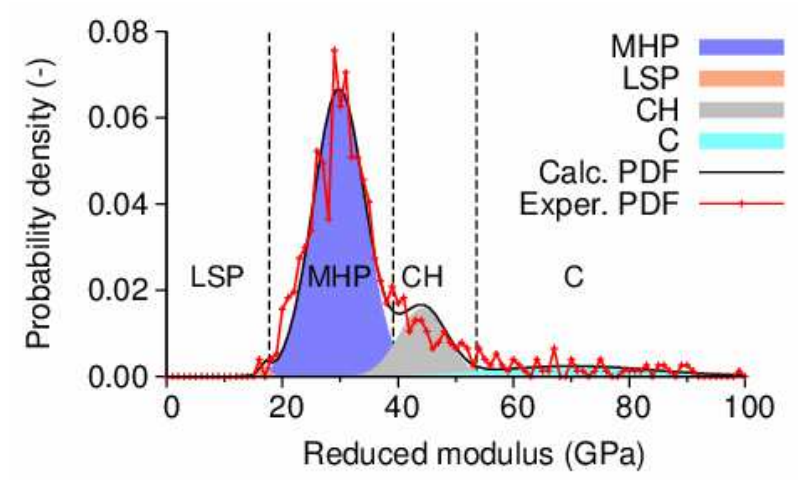

Figure 2: Deconvolution of reduced moduli in cement paste. 
The red curve in Fig. 2 belongs to experimental points whereas black dashed line is a theoretical fit received from the deconvolution. The dotted lines in Fig. 2 represent intersections of the probability density functions (PDF) between the neighboring distributions. The intersecting points were used for separation of indents into groups belonging to individual microstructural phases.

\subsection{Fracture surface}

Selected indents were sectioned by FIB and inspected for crack position, depth and inclination with respect to the surface. The situation is shown in Fig. 3 and 4. It is clearly seen in the figures that cracks are located in the perimeter of an indent. These surface cracks propagate downwards to the material in the perpendicular direction to the indentation depression, i.e. approximately 65.3 degrees with respect to the surface. The crack inclination is dictated by the tip geometry and the evolution of high tensional stresses caused by the material stretching that appears around the indent in a perpendicular direction to the crack. As observed by FIB the crack depth is approximately equivalent to the indentation (contact) depth (Fig. 4).

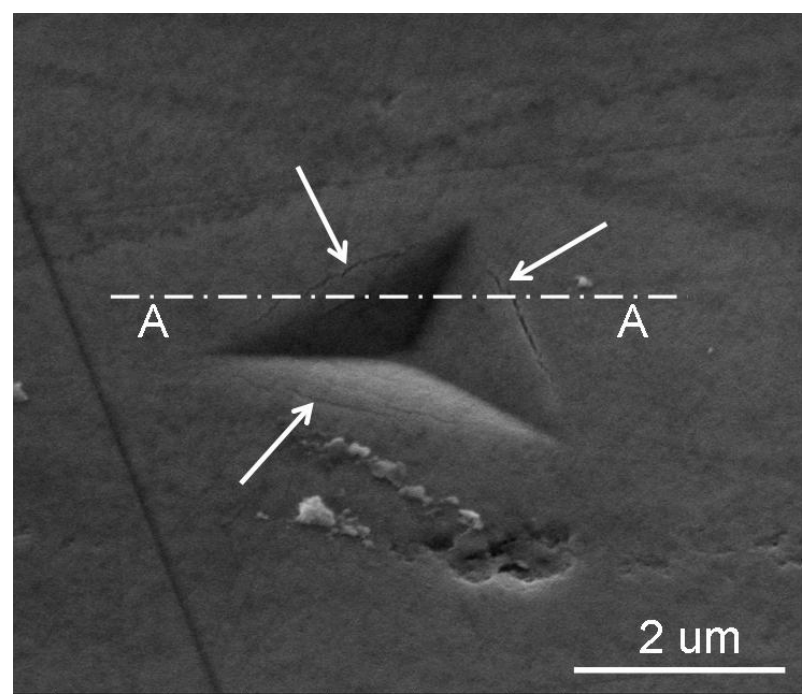

Figure 3: An indent before sectioning. Cracks located around the indent are marked by arrows. The dash-anddotted line indicates position of a cross section shown in the next figure (Fig. 4).

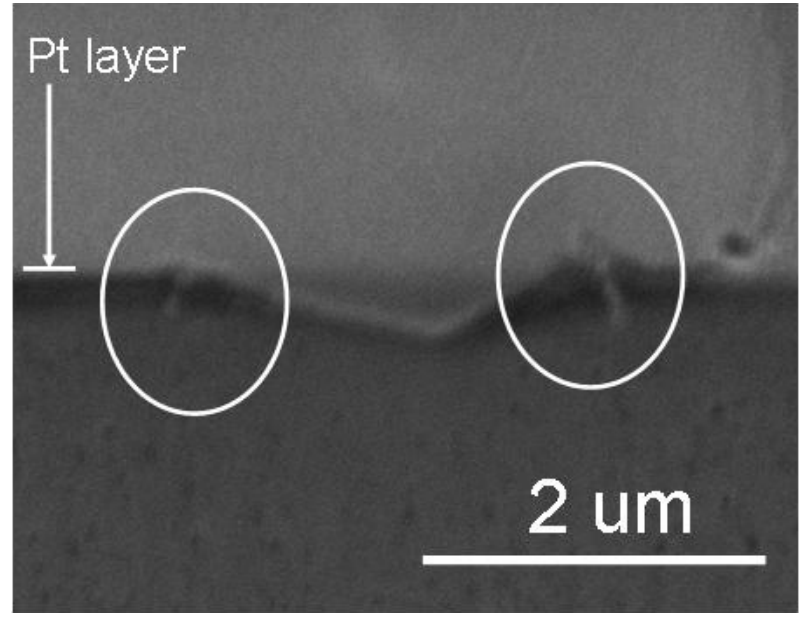

Figure 4: Cross section of an indent (indicated by dashand-dotted line in Fig. 3). Cracks are highlighted in circles. To improve contrast during SEM observation, the surface is covered by Pt layer.

Frequently, more than one crack (typically two) parallel with a major one is located around an indent. Based on these findings, the minimum fracture area can be estimated to have a doubled surface of truncated pyramid sides with the height equal to the indentation depth and the wall inclination 65.3 degrees as illustrated in Fig. 5.

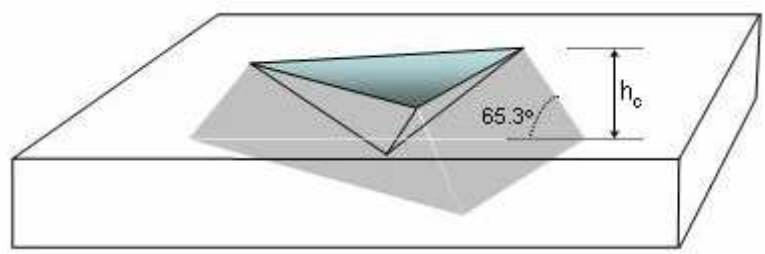

Figure 5: Assumption on the fracture area around an indent (grey inclined surface).

It is worth mentioning that the findings shown here about the fracture area differ significantly from the assumption used by other authors who estimated the fracture area in cement e.g. as the tip contact area [10]. It is shown here that such estimates are unphysical and the knowledge delivered by FIB is inevitable for this purpose.

\subsection{Fracture energy and fracture toughness}

Once, the assumption on the fracture area is done, calculation of fracture energies can be performed using Eqs. 2, 3 and 4. It must be emphasized that the same assumption on the fracture area from section 3.2 (depending on 
the contact depth) was used for all indents regardless their position and phase indent. An exact calculation would require FIB sectioning of every indent which is not feasible. Therefore, the results must be treated as semiquantitative in this sense. Further, the exact fracture area can involve more than one or two major parallel cracks around the indent. Tracking of some minor cracks that contribute to the energy release can be lost due to the resolution limits of SEM imaging. The pixel size corresponds to $7.3 \mathrm{~nm}$ in Figs. 3 and 4. The major crack width is about $50 \mathrm{~nm}$ for this case. The presence of much smaller (nondetectable) cracks cannot be fully excluded. Thus, the calculated fracture energy presented here represents the best upper estimate of the energy for a given phase. The linear-elastic stress intensity factor (fracture toughness) can be calculated from the fracture energy for plane strain conditions as

$$
K_{C}=\sqrt{E_{r} G_{c}}
$$

Note, that $E_{r}=E /\left(1-v^{2}\right)$ where $E$ is Young's modulus and $v$ is Poisson's ratio.

The overall results of $G_{c}$ and $K_{C}$ are summarized in Tab. 1 where LSP stands for low stiffness phases, $\mathrm{MHP}=$ main hydration products, $\mathrm{CH}=$ calcium hydroxide and $\mathrm{C}=$ clinker.

Table 1: Mechanical results for distinct microscale phases $\left(E_{r}=\right.$ reduced modulus, $f=$ volume fraction, $G_{c}^{u p p}=$ critical energy release rate, $K_{c}^{\text {upp }}=$ fracture toughness).

\begin{tabular}{lcccc}
\hline Phase & $\begin{array}{c}E_{r} \\
(\mathrm{GPa})\end{array}$ & $f$ & $\begin{array}{c}G_{c}^{\text {upp }} \\
\left(\mathrm{J} / \mathrm{m}^{2}\right)\end{array}$ & $\begin{array}{c}K_{c}^{\text {upp }} \\
\left(\mathrm{MPam}^{1 / 2}\right)\end{array}$ \\
\hline LSP & $17.0 \pm 0.8$ & 0.005 & $16.0 \pm 2.2$ & $0.51 \pm 0.03$ \\
\hline MHP & $29.8 \pm 4.5$ & 0.747 & $16.7 \pm 6.2$ & $0.70 \pm 0.15$ \\
\hline CH & $44.2 \pm 4.1$ & 0.163 & $25.2 \pm 10.9$ & $1.04 \pm 0.24$ \\
\hline C & $70.3 \pm 14.0$ & 0.085 & $42.8 \pm 22.6$ & $1.69 \pm 0.55$ \\
\hline
\end{tabular}

It can be seen from Tab. 1 that the majority of the sample volume $(74.7 \%)$ is composed of main hydration products. The upper estimate for the fracture energy for this phase was calculated as $16.7 \pm 6.2 \mathrm{~J} / \mathrm{m}^{2}$ and the related fracture toughness as $0.70 \pm 0.15 \mathrm{MPam}^{1 / 2}$. The low-scale fracture toughness is related (and a bit higher) to macroscopic values that for cement past usually take the values $0.3-0.5$ $\mathrm{MPam}^{1 / 2}$. Note, that a small number of occurances was captured for low stiffness phases (only 5\% represented by 3 indents) and the results are thus not representative enough. On the other hand, the fracture toughness of calcium hydroxide as well as clinker minerals is much higher, larger than $1 \mathrm{MPam}^{1 / 2}$. Since the volumetric content of $\mathrm{CH}$ and $\mathrm{C}$ phases in the paste is not negligible $(16.3 \%+8.5 \%)$ it can be deduced that these phases contribute to the overall paste fracture toughness also by nonnegligible contributions. It follows from theoretical works (e.g. Ghebrab and Soroushian [11]) that $\mathrm{CH}$ crystal pull-out raises the values of pure $\mathrm{C}-\mathrm{S}-\mathrm{H}$ to much higher values, $16.9-283 \mathrm{~J} / \mathrm{m}^{2}$, which is in relation to our findings, whereas the pure $\mathrm{C}-\mathrm{S}-\mathrm{H}$ fracture energy ranges only between $0.38-2.85 \mathrm{~J} / \mathrm{m}^{2}$ $[11,12]$.

Also, the reduced modulus received from nanoindentation for clinker is underestimated due to high stiffness contrast to the surrounding compliant matrix. Although corrections can be done [1] they were not applied in Tab. 1. Therefore, it can be expected that the clinker fracture toughness is even higher. Assuming the real clinker value of $E_{r}=126 \mathrm{GPa}$ [1], the average clinker fracture toughness equals to $2.32 \mathrm{MPam}^{1 / 2}$.

\section{CONCLUSIONS}

The paper presents the way how to identify elastic and fracture parameters of low-scale cement paste phases using a combination of advanced experimental techniques. The primary role in the identification plays nanoindentation as a mechanical tool that is capable to quantitatively measure load-depth diagrams in the order of hundreds of nanometres (in our case) for individual material phases. Focused ion beam is capable of disclosing fracture phenomena and helps to quantify assumptions on size, shape and extent of a fracture area underneath the surface. Based on the simplified assumptions derived from FIB analysis it was possible to calculate upper estimates of the fracture energy and 
fracture toughness for individual hydrated cement components on a micrometer scale. The values obtained in this work relate to the macroscopic values and are in range of theoretical predictions derived on a molecular level of C-S-H.

\section{Acknowledgements}

Financial support of the Czech Science Foundation (project 16-11879S) and the Grant Agency of the Czech Technical University in Prague (OHK1-074/16) is gratefully acknowledged.

\section{REFERENCES}

[1] Němeček, J., Králík, V., Vondřejc, J. 2013. Micromechanical analysis of heterogeneous structural materials, $\mathrm{Cem}$ Concr Comp 36, 85-92.

[2] da Silva, W. R. L.; Nemecek, J. 2014. Stemberk, P., Methodology for nanoindentation-assisted prediction of macroscale elastic properties of high performance cementitious composites, Cem Concr Comp 45, 57-68.

[3] Minster, J.; Blahova, O.; Lukes, J.; et al. 2010. Time-dependent mechanical characteristics measured through the use of a microindentation technique, Mech Time-Dep Mat 14 (3), 243-251.

[4] Cook, R.F., Pharr, G.M. 1990. Direct observation and analysis of indentation cracking in glasses and ceramics. J. Am. Ceram. Soc. 73, 787-817.

[5] Lawn, B.R., Evans, A.G., Marshall, D.B. 1980. Elastic/plastic indentation damage in ceramics: the median/radial crack system. J. Am. Ceram. Soc. 63, 574-581.

[6] Chen, J., Bull, S.J. 2007. Indentation fracture and toughness assessment for thin optical coatings on glass. J. Phys. D-Appl. Phys. 40, 5401-5417.

[7] Cheng, Y.T., Li, Z.Y., Cheng, C.M. 2002. Scaling relationships for indentation measurements, Philos. Mag. A 82, 18211829.

[8] Oliver, W., Pharr, G. 1992. An Improved Technique for Determining Hardness and Elastic-Modulus using Load and
Displacement Sensing Indentation Experiments, J. Mater. Res. 7, 1564-1583.

[9] Yeakub, A.M., Wayne, H., Fu, Y. 2010. A review of focused ion beam sputtering. International Journal of Precision Engineering and Manufacturing 11 (1), 157-170.

[10]Taha, R.M., et al. 2010. Fracture toughness of hydrated cement paste using nanoindentation, Fracture Mechanics of Concrete and Concrete Structures Recent Advances in Fracture Mechanics of Concrete - B. H. Oh, et al.(eds).

[11] Ghebrab, T., Soroushian, P. 2010. Mechanical properties of hydrated cement paste: Development of structure-property relationships, Journal of Concrete Structures and Materials 7 (4), 37-43.

[12]Bauchy, M., Laubie, H., Qomi, M. A. , Hoover, C., Ulm, F.-J., Pellenq, R.-M. 2015. Fracture toughness of calciumsilicate-hydrate from molecular dynamics simulations, Journal of Non-Crystalline Solids 419, 58-64. 